\title{
Protection of Aroma Volatiles in a Red Wine with Low Sulphur Dioxide by a Mixture of Glutathione, Caffeic Acid and Gallic Acid
}

\author{
I.G. Roussis ${ }^{1 *}$, M. Patrianakou ${ }^{1}$ and A. Drossiadis ${ }^{2}$ \\ (1) Laboratory of Food Chemistry, Department of Chemistry, University of Ioannina, 45110 Ioannina, Greece \\ (2) Oenoforos Winery, 25100 Egio, Greece
}

Submitted for publication: March 2013

Accepted for publication: August 2013

Key words: Red wine, volatiles, sulphur dioxide, glutathione, caffeic acid, gallic acid

\begin{abstract}
The levels of several esters and other volatiles in Merlot-Cabernet Sauvignon blend red wines with typical sulphur dioxide (35 mg/L), with low sulphur dioxide $(25 \mathrm{mg} / \mathrm{L})$, and with low sulphur dioxide (25 mg/L) plus a mixture of antioxidants (glutathione $20 \mathrm{mg} / \mathrm{L}$, caffeic acid $60 \mathrm{mg} / \mathrm{L}$ and gallic acid $20 \mathrm{mg} / \mathrm{L}$ ), were evaluated at bottling, and after 18 and 36 months of ageing. Most volatiles decreased during wine storage. At bottling and after 18 months of storage, all three wines exhibited similar levels of volatiles. After 36 months of storage, wines with low sulphur dioxide exhibited lower levels of many volatiles, such as ethyl acetate, ethyl hexanoate, ethyl octanoate and 2-phenylethanol. On the other hand, wines with low sulphur dioxide plus the mixture of antioxidants exhibited similar levels of most volatiles in comparison with wines with typical sulphur dioxide. The present results indicate that a mixture of glutathione, caffeic acid and gallic acid can protect esters and other volatiles in young red wine with low sulphur dioxide, and can replace part of sulphur dioxide typically used.
\end{abstract}

\section{INTRODUCTION}

Oxidative spoilage of both white and red wines is a wellknown problem in winemaking. The first step of oxidation is characterised by the transformation of aroma compounds, leading to a loss of characteristic wine aromas and, subsequently, to the formation of new aromas characteristic of older wines or atypical aromas associated with wine deterioration (Singleton, 1987; Vaimakis \& Roussis, 1996; Ferreira et al., 1997; Roussis et al., 2005).

Sulphur dioxide is the most common preservative used in winemaking, exhibiting both antioxidant and antimicrobial properties. However, when free sulphur dioxide levels are between 15 and $40 \mathrm{mg} / \mathrm{L}$, most individuals begin to detect a distinctive burnt match odour. Moreover, the consumption of high concentrations of sulphites may have adverse health effects on humans, such as giving rise to asthma. As a result of these disadvantages of sulphur dioxide addition to wine, the trend is to limit its use.

As far as wine aroma compounds are concerned, it has been reported that sulphur dioxide protects several aroma volatiles, such as esters, alcohols and fatty acids, during wine ageing (Garde-Cerdan \& Ancin-Azpilicueta, 2007; Roussis et al., 2007; Roussis \& Sergiantitis, 2008).

The antioxidant tripeptide glutathione is a natural constituent, and the main thiol, of wines. Phenolics are major wine antioxidants. Among them, caffeic acid is the main hydroxycinnamic acid and gallic acid the main (hydroxy) benzoic acid in wines. Regarding wine aroma compounds, it has been found previously that glutathione, caffeic acid, gallic acid, and also wine phenolic extracts slow the decrease of several esters and terpenes during white wine storage (Roussis et al., 2005; Lambropoulos \& Roussis, 2007a, 2007b; Papadopoulou \& Roussis, 2008), and glutathione slows the decrease of volatile thiols during the storage of Sauvignon wine (Dubourdieu \& Lavigne-Cruege, 2004). Moreover, it has been observed that the addition of a mixture of glutathione and caffeic acid to a white wine containing low free sulphur dioxide has a protective effect on volatile esters and terpenes (Roussis et al., 2007).

In the present study, we investigated the ability of a mixture of glutathione, caffeic acid and gallic acid to protect aroma volatiles during the storage of a red wine with low sulphur dioxide.

\section{MATERIALS AND METHODS}

Glutathione 98\%, caffeic acid 98\% and gallic acid 97\%, as well as the absolute ethanol used, were purchased from Sigma (St. Louis, MO, USA). A Merlot-Cabernet Sauvignon $(60-40)$ blend red wine produced in the industry was used; the producers recommend that the wine is consumed within three years after production. Cryoextraction was applied during winemaking, and decantations were applied after alcoholic and malolactic fermentation. Micro-oxygenation was applied to the young wines for about three months; the

*Corresponding author: E-mail: iroussis@uoi.gr

Aknowledgements: The GC-MS facilities of the Food Quality Certification Unit of the University of Ioannina were used for this work 
delivered quantity of oxygen was $2.5 \mathrm{mg} / \mathrm{L}$ of wine per week. After clarification and stabilisation, the wine was bottled in bottles of $750 \mathrm{~mL}$, using natural corks for moderate ageing (Alves Portugal Cork).

In the control wines, sulphur dioxide was added at bottling and the levels of sulphur dioxide were typical, i.e. $35 \mathrm{mg} / \mathrm{L}$ free sulphur dioxide and $75 \mathrm{mg} / \mathrm{L}$ total sulphur dioxide. In the red wine with low sulphur dioxide, no addition of sulphur dioxide was done at bottling. Sulphur dioxide levels were $25 \mathrm{mg} / \mathrm{L}$ free sulphur dioxide and $58 \mathrm{mg} / \mathrm{L}$ total sulphur dioxide. The third experimental wine was the wine with low sulphur dioxide to which the mixture of glutathione, caffeic acid and gallic acid was added at bottling. For this, $5 \mathrm{~mL}$ was removed per $1000 \mathrm{~mL}$ of wine, and $5 \mathrm{~mL}$ of $50 \%$ ethanol containing glutathione, caffeic acid and gallic acid was added in order for the final concentration of the three antioxidants in the wine to be $20 \mathrm{mg} / \mathrm{L}, 60 \mathrm{mg} / \mathrm{L}$ and $20 \mathrm{mg} / \mathrm{L}$ respectively. In the control wine and the wine with low sulphur dioxide, $5 \mathrm{~mL}$ of wine were removed per $1000 \mathrm{~mL}$ and $5 \mathrm{~mL}$ of $50 \%$ ethanol were added.

The gross composition of the red wine at bottling was as follows: alcohol content $13.0 \%$ vol, residual sugars $1.4 \mathrm{~g} / \mathrm{L}$, $\mathrm{pH} 3.46$, total acidity $5.6 \mathrm{~g} / \mathrm{L}$ as tartaric acid, and volatile acidity $0.3 \mathrm{~g} / \mathrm{L}$ as acetic acid. Alcohol was determined with a hydrometer, reducing sugars were determined by the Lane-Eynon method, $\mathrm{pH}$ with a $\mathrm{pH}$ meter, total acidity by volumetric analysis, and volatile acidity by steam distillation. Total and free sulphur dioxide was determined by the Ripper method. The bottles were stored in a dark room at $18^{\circ} \mathrm{C}$. After 0,18 and 36 months of storage, bottles were taken and wine samples were analysed for volatiles. All wine samples were analysed by solid phase microextraction (SPME) along with gas chromatography-mass spectrometry (GC-MS) (Vas \& Vekey, 2004; Castro et al., 2008). A polydimethylsiloxane 100 um fibre (Supelco, Bellefonte, PA, USA) was used for the absorption of volatiles.

Two $\mathrm{mL}$ of each wine sample and $50 \mu \mathrm{L}$ of internal standard in 10\% ethanol (4-methyl-1-pentanol, $5 \mathrm{mg} / \mathrm{L}$ in final solution) were transferred to a $4 \mathrm{~mL}$ screw-capped glass vial with a Teflon-rubber septum (red, $12 \mathrm{~mm}$, SunSri, Rockwood, TN, USA). The contents were stirred for $15 \mathrm{~min}$ at $40^{\circ} \mathrm{C}$, after which a constant length of the fibre was exposed to the headspace for another $20 \mathrm{~min}$ at $40^{\circ} \mathrm{C}$ without stirring. Desorption of volatiles took place at $250^{\circ} \mathrm{C}$ using a $0.75 \mathrm{~mm}$ ID liner (Supelco, Bellefonte, PA, USA) for $5 \mathrm{~min}$. Split mode was used, and the split ratio was $2: 1$. GC-MS analysis was carried out on an HP 5973 quadrupole mass spectrometer directly coupled to an HP 6890 gas chromatograph.

MS conditions were as follows: source temperature: $230^{\circ} \mathrm{C}$, quadrupole temperature: $150^{\circ} \mathrm{C}$, transfer line temperature: $270^{\circ} \mathrm{C}$. Acquisition was performed in electron impact (EI) mode $(70 \mathrm{eV})$ by $2.02 \mathrm{scans} \mathrm{s}^{-1}$, and the mass range was 29 to $400 \mathrm{~m} / \mathrm{z}$. Solvent delay was applied up to 6.0 min to avoid the ethanol peak. A DB-5 fusedsilica capillary column was used $(60 \mathrm{~m}$ x $0.32 \mathrm{~mm}$ internal diameter, $1.0 \mu \mathrm{m}$ film thickness, J\&W Scientific, Folsom, CA, USA). The flow rate of the helium carrier gas was $1.1 \mathrm{~mL} \mathrm{~min}{ }^{-1}$. The oven temperature was programmed to start at $40^{\circ} \mathrm{C}$ for $5 \mathrm{~min}$, and then raised to $260^{\circ} \mathrm{C}$ at a rate of $5^{\circ} \mathrm{C} / \mathrm{min}$, and it was held at $260^{\circ} \mathrm{C}$ for $10 \mathrm{~min}$. All peaks were identified by comparing mass spectra to those obtained from the Wiley library (Wiley 275; J. Wiley \& Sons Ltd., West Sussex, England).

The identification of many peaks was confirmed with mass spectra and retention times of standard compounds determined under the same analysis conditions. Authentic standards used were ethyl acetate 99\%, isoamyl acetate 99\%, n-hexyl acetate 99\%, 2-phenyl ethyl acetate 99\%, ethyl butanoate $99 \%$, ethyl hexanoate $99 \%$, ethyl octanoate $99 \%$, ethyl decanoate $99 \%$, ethyl dodecanoate $99 \%$, 1-hexanol 98\% (Merck, Darmstadt, Germany), hexanoic acid 98\%, octanoic acid 98\%, decanoic acid 98\%, and 4-methyl-pentanol 99\% (Sigma, St. Louis, MO, USA). Semiquantitative data were expressed in milligrams per litre [(area of compound/area of internal standard) $\cdot$ concentration of internal standard]. The whole experiment was repeated three times and the results reported are the means of the three trials. The one-way analysis of variance (ANOVA), using the Bonferroni test at a level of significance of $\mathrm{P}<0.05$, was used for statistical analysis (SPSS 17.0) (SPSS Inc., Chicago, IL).

\section{RESULTS AND DISCUSSION}

The levels of volatiles of a young Merlot-Cabernet Sauvignon blend wine a) with typical sulphur dioxide (35 mg/L), designated as TSD wine, b) with low sulphur dioxide (25 mg/L), designated as LSD wine, and c) with low sulphur dioxide $(25 \mathrm{mg} / \mathrm{L})$ plus a mixture of antioxidants (glutathione $20 \mathrm{mg} / \mathrm{L}$, caffeic acid $60 \mathrm{mg} / \mathrm{L}$ and gallic acid $20 \mathrm{mg} / \mathrm{L}$ ), designated as LSDA wine, were evaluated during wine storage (Table 1).

Most volatiles decreased during the storage of TSD wine. These are the esters isoamyl acetate, 5-methyl-3-heptanoate, ethyl hexanoate, n-hexyl acetate, ethyl octanoate, 2-phenyl ethyl acetate, ethyl decanoate and ethyl dodecanoate, the alcohols 1-hexanol and 2-phenylethanol, the polyols 1,3-butanediol and 2,3-butanediol, and the fatty acids hexanoic acid, octanoic acid and decanoic acid. However, some other volatiles, particularly ethyl acetate and also ethyl butanoate and ethyl 9-decanoate, were stable during wine storage. A decrease of most esters, alcohols and fatty acids during the storage of white and red wines has been reported by others (Perez-Prieto et al., 2003; Roussis et al., 2007).

At bottling ( 0 months) and after 18 months of storage, the LSD and LSDA wines exhibited similar levels of volatiles compared to the TSD wine. These results indicate that red wines with low sulphur dioxide exhibited similar levels of volatiles during storage for a significant period. Moreover, the results indicate that the addition of the mixture of antioxidants did not change the levels of volatiles during wine storage for a significant period.

After 36 months of storage, the LSD wine exhibited lower levels of many volatiles - esters, fatty acids and alcohols - compared to the TSD wine. Among them were ethyl acetate, isoamyl acetate, 5-methyl-3-heptanoate, ethyl hexanoate, ethyl octanoate, 1-hexanol, 2-phenylethanol, 1,3-butanediol and hexanoic acid. These results indicate that the addition of sulphur dioxide at bottling and the control of its concentration at typical levels $(35 \mathrm{mg} / \mathrm{L})$ protected these volatiles during long storage of the wine. The protection of 
TABLE 1

Relative concentrations (mg/L) of volatiles in experimental Merlot-Cabernet Sauvignon red wines during storage.

\begin{tabular}{llllll}
\hline Volatiles & TSD wine & TSD wine & TSD wine & LSD wine & LSDA \\
& 0 months & 18 months & 36 months & 36 months & 36 months \\
\hline Ethyl acetate & $20.3^{\mathrm{A}} \pm 2.7$ & $21.4^{\mathrm{A}} \pm 1.5$ & $23.1^{\mathrm{Aa}} \pm 1.9$ & $9.8^{\mathrm{b}} \pm 1.0$ & $22.5^{\mathrm{a}} \pm 1.1$ \\
Ethyl butanoate & $1.1^{\mathrm{A}} \pm 0.1$ & $1.0^{\mathrm{A}} \pm 0.1$ & $0.7^{\mathrm{Aa}} \pm 0.1$ & $0.5^{\mathrm{b}} \pm 0.0$ & $0.8^{\mathrm{a}} \pm 0.0$ \\
Isoamyl acetate & $9.6^{\mathrm{A}} \pm 1.5$ & $4.1^{\mathrm{B}} \pm 0.3$ & $1.5^{\mathrm{Ca}} \pm 0.2$ & $1.1^{\mathrm{b}} \pm 0.1$ & $1.4^{\mathrm{a}} \pm 0.1$ \\
5-methyl-3-heptanoate & $7.2^{\mathrm{A}} \pm 0.1$ & $4.7^{\mathrm{B}} \pm 0.3$ & $3.5^{\mathrm{Ba}} \pm 0.5$ & $2.5^{\mathrm{b}} \pm 0.09$ & $3.4^{\mathrm{a}} \pm 0.2$ \\
Ethyl hexanoate & $28.3^{\mathrm{A}} \pm 3.0$ & $21.8^{\mathrm{B}} \pm 1.9$ & $12.6^{\mathrm{Ba}} \pm 0.2$ & $10.8^{\mathrm{b}} \pm 0.6$ & $14.4^{\mathrm{a}} \pm 1.0$ \\
n-hexyl acetate & $1.4^{\mathrm{A}} \pm 0.2$ & $0.4^{\mathrm{B}} \pm 0.1$ & 0.0 & 0.0 & 0.0 \\
Ethyl octanoate & $138.1^{\mathrm{A}} \pm 18.0$ & $95.6^{\mathrm{B}} \pm 8.6$ & $48.6^{\mathrm{b}} \pm 1.1$ & $40.5^{\mathrm{c}} \pm 1.5$ & $60.7^{\mathrm{a}} \pm 1.4$ \\
2-phenyl ethyl acetate & $1.8^{\mathrm{A}} \pm 0.3$ & $0.9^{\mathrm{B}} \pm 0.1$ & $0.3^{\mathrm{Aa}} \pm 0.1$ & $0.2^{\mathrm{a}} \pm 0.1$ & $0.3^{\mathrm{a}} \pm 0.1$ \\
Ethyl decanoate & $57.7^{\mathrm{A}} \pm 10.4$ & $34.2^{\mathrm{B}} \pm 1.6$ & $13.2^{\mathrm{Cb}} \pm 1.0$ & $12.9^{\mathrm{b}} \pm 1.5$ & $17.1^{\mathrm{a}} \pm 0.5$ \\
Ethyl dodecanoate & $2.6^{\mathrm{A}} \pm 0.4$ & $2.2^{\mathrm{A}} \pm 0.4$ & $0.6^{\mathrm{Ba}} \pm 0.1$ & $0.5^{\mathrm{a}} \pm 0.2$ & $0.7^{\mathrm{a}} \pm 0.1$ \\
1,3-butanediol & $9.2^{\mathrm{A}} \pm 1.3$ & $7.6^{\mathrm{A}} \pm 0.5$ & $3.4^{\mathrm{Ba}} \pm 0.4$ & $2.1^{\mathrm{b}} \pm 0.5$ & $3.9^{\mathrm{a}} \pm 0.6$ \\
2,3-butanediol & $1.7^{\mathrm{A}} \pm 0.2$ & $1.3^{\mathrm{B}} \pm 0.1$ & $0.5^{\mathrm{Cb}} \pm 0.2$ & $0.4^{\mathrm{b}} \pm 0.2$ & $0.9^{\mathrm{a}} \pm 0.1$ \\
1-hexanol & $6.5^{\mathrm{A}} \pm 0.4$ & $4.2^{\mathrm{B}} \pm 0.2$ & $3.1^{\mathrm{Ca}} \pm 0.1$ & $2.2^{\mathrm{b}} \pm 0.1$ & $3.1^{\mathrm{a}} \pm 0.2$ \\
2-phenylethanol & $64.8^{\mathrm{A}} \pm 14.6$ & $43.9^{\mathrm{B}} \pm 4.2$ & $26.1^{\mathrm{Ca}} \pm 1.0$ & $19.1^{\mathrm{b}} \pm 1.4$ & $29.1^{\mathrm{a}} \pm 1.7$ \\
Hexanoic acid & $3.6^{\mathrm{A}} \pm 0.4$ & $2.6^{\mathrm{A}} \pm 0.2$ & $1.1^{\mathrm{Ba}} \pm 0.2$ & $0.6^{\mathrm{b}} \pm 0.1$ & $0.6^{\mathrm{b}} \pm 0.1$ \\
Octanoic acid & $7.0^{\mathrm{A}} \pm 2.0$ & $7.4^{\mathrm{A}} \pm 1.0$ & $3.4^{\mathrm{Ba}} \pm 0.3$ & $2.4^{\mathrm{a}} \pm 0.8$ & $3.4^{\mathrm{a}} \pm 0.2$ \\
Decanoic acid & $1.8^{\mathrm{A}} \pm 0.8$ & $1.9^{\mathrm{A}} \pm 0.3$ & $0.5^{\mathrm{Ba}} \pm 0.1$ & $0.5^{\mathrm{a}} \pm 0.2$ & $0.5^{\mathrm{a}} \pm 0.1$ \\
\hline
\end{tabular}

TSD wine: wine with typical sulphur dioxide (35 mg/L); LSD wine: wine with low sulphur dioxide (25 mg/L); LSDA wine: wine with low sulphur dioxide $(25 \mathrm{mg} / \mathrm{L}$ ) plus a mixture of antioxidants (glutathione $20 \mathrm{mg} / \mathrm{L}$, caffeic acid $60 \mathrm{mg} / \mathrm{L}$ and gallic acid $20 \mathrm{mg} / \mathrm{L}$ ).

Values in $\mathrm{mg} / \mathrm{L}$ as 4-methyl-1-pentanol are the means of three trials along with standard deviations.

Different capital letters indicate significant differences among TSD wine samples stored for 0, 18 and 36 months.

Different lowercase letters indicate significant differences among TSD, LSD and LSDA wine samples after 36 months of storage.

aroma volatiles - esters, alcohols and fatty acids - by sulphur dioxide during wine storage has been reported by others (Garde-Cerdan \& Ancin-Azpilicueta, 2007; Roussis et al., 2007; Roussis \& Sergianitis, 2008).

After 36 months of storage, the LSDA wine exhibited higher levels of most volatiles in comparison to the LSD wine. These volatiles were ethyl acetate, ethyl butanoate, isoamyl acetate, 5-methyl-3-heptanoate, ethyl hexanoate, ethyl octanoate, ethyl 9-decanoate, ethyl decanoate, 1-hexanol, 2-phenylethanol, 1,3-butanediol and 2,3-butanediol. These results indicate that the addition of the mixture of antioxidants to red wine with low sulphur dioxide protected these volatiles during long storage of the wine.

After 36 months, the LSDA and TSD wines exhibited similar levels of most volatiles, such as ethyl acetate, ethyl butanoate, isoamyl acetate, 5-methyl-3-heptanoate, ethyl hexanoate, ethyl 9-decanoate, 1-hexanol, 2-phenylethanol and 1,3-butanediol. Ethyl octanoate, ethyl decanoate and 2,3-butanediol were higher in the LSDA wine, and hexanoic acid in the TSD wine. These results indicate that the addition of the mixture of antioxidants to red wine with low sulphur dioxide $(25 \mathrm{mg} / \mathrm{L})$ at bottling replaced the addition of sulphur dioxide and its control at typical levels $(35 \mathrm{mg} / \mathrm{L})$ as far as the protection of aroma volatiles is concerned.

The Merlot-Cabernet Sauvignon blend wine studied is a red wine with floral and fruity notes recommended for consumption within three years. As indicated by two of the judges, the wine with low sulphur dioxide exhibited lower sensory quality, aroma and taste than the control wine. On the other hand, the wine with low sulphur dioxide plus the mixture of antioxidants retained its sensory quality and was comparable to the wine with typical sulphur dioxide.

Several chemical reactions influence the composition of the volatile constituents of wine during ageing. Ester changes can be due to hydrolysis and esterification phenomena (Ramey \& Ough, 1980), and also to their oxidation initiated by hydroxyl radicals (Patrianakou \& Roussis, 2013). Changes in the concentration of fatty acids and alcohols may be explained by their generation due to the hydrolysis of esters and by their oxidation to yield aldehydes (Nykanen, 1986; Perez-Prieto et al., 2003). Subsequently, the protective effect of sulphur dioxide and also of glutathione, caffeic acid and gallic acid may be attributed to their antioxidant properties.

A significant part of the basic odour of wines is attributed to the esters ethyl acetate, isoamyl acetate, ethyl hexanoate and ethyl octanoate. Acetates and ethyl esters are important contributors to the aroma of young wine and they exhibit floral and fruity odours. Alcohols have intense odours that play a role in wine aroma, while fatty acids contribute a fresh flavour to wine (Rapp \& Mandery, 1986). Acetates and ethyl esters, 2-phenylethanol, 1-hexanol and butanediols, and hexanoic and octanoic acids have been reported as significant aroma volatiles of Merlot and Cabernet Sauvignon wines 
(Ferreira et al., 2000; Kotseridis \& Baumes, 2000; Tao \& Li, 2009; Welke et al., 2012).

Taking the above into account, the present results indicate that the control of sulphur dioxide, and alternatively the increase in the concentrations of glutathione, caffeic acid and gallic acid along with low sulphur dioxide, are critical for the retention of aroma volatiles during the storage of red wines exhibiting floral and fruity aroma, such as the MerlotCabernet Sauvignon blend used in this study.

\section{CONCLUSIONS}

The present results indicate that a mixture of glutathione, caffeic acid and gallic acid can protect esters and other volatiles in red wine with a low sulphur dioxide content and can replace part of the sulphur dioxide typically used.

\section{LITERATURE CITED}

Castro, R., Natera, R., Durán, E. \& García-Barroso, C., 2008. Application of solid phase extraction techniques to analyse volatile compounds in wines and other enological products. Eur. Food Res. Technol. 228, 1-18.

Dubourdieu, D. \& Lavigne-Cruege, V., 2004. The role of glutathione on the aromatic evolution of dry white wine. Vinidea Net Wine Internet Techol. J. 2, 1-9.

Ferreira, V., Escudero, A., Fernadez, P. \& Cacho, J.F., 1997. Changes in the profile of volatile compounds in wines stored under oxygen and their relationship with the browning process. Z. Lebensm.-Unters.-Forsch. 205, 392-396.

Ferreira, V., Lopez, R. \& Cacho, J.F., 2000. Quantitative determination of the odorants of young red wines from different grape varieties. J. Sci. Food Agric. 80, 1659-1667.

Garde-Cerdan, T. \& Ancin-Azpilicueta, C., 2007. Effect of $\mathrm{SO}_{2}$ on the formation and evolution of volatile compounds in wines. Food Control 18, 1501-1506.

Kotseridis, Y. \& Baumes, R., 2000. Identification of impact odorants in Bordeaux red grape juice, in the commercial yeast used for its fermentation, and in the produced wine. J. Agric. Food Chem. 48, 400-406.

Lambropoulos, I. \& Roussis, I.G., 2007a. Inhibition of the decrease of volatile esters and terpenes during storage of a white wine and a model wine medium by caffeic acid and gallic acid. Food Res. Intern. 40, 176-181.

Lambropoulos, I. \& Roussis, I.G., 2007b. Inhibition of the decrease of volatile esters and terpenes during storage of wines and a model wine medium by wine phenolic extracts. Food Techn. Biotechn. 45, 147-155.
Nykanen, L., 1986. Formation and occurrence of flavor compounds in wine and distilled alcoholic beverages. Am. J. Enol. Vitic. 36, 170-174.

Papadopoulos, D. \& Roussis, I.G., 2008. Inhibition of the decrease of volatile esters and terpenes during storage of a white wine and a model wine medium by glutathione and $N$-acetyl-cysteine. Intern. J. Food Sci. Technol. 43, 1053-1057.

Patrianakou, M. \& and Roussis, I.G., 2013. Decrease of wine volatile aroma esters by oxidation. S. Afr. J. Enol. Vitic. 34, 241-245.

Perez-Prieto, L.J., Lopez-Roca, J.M. \&. Gomez-Plaza, E., 2003. Differences in major volatile compounds of red wine according to storage length and storage conditions. J. Food Comp. Anal. 16, 697-705.

Ramey, D.D. \& Ough, C.S., 1980. Volatile ester hydrolysis or formation during storage of model solutions and wines. J. Agric. Food Chem. 28, 928934.

Rapp, A. \& Mandery, H., 1986. Wine aroma. Experimentia 42, 873-884.

Roussis, I.G. \& Sergianitis, S., 2008. Protection of some aroma volatiles in a model wine medium by sulphur dioxide and mixtures of glutathione with caffeic acid or gallic acid. Flav. Fragr. J. 23, 35-39.

Roussis, I.G., Lambropoulos, I. \& Papadopoulou, D., 2005. Inhibition of the decline of volatile esters and terpenols during oxidative storage of Muscatwhite and Xinomavro-red wine by caffeic acid and $\mathrm{N}$-acetyl-cysteine. Food Chem. 93, 485-492.

Roussis, I.G., Lambropoulos, I. \& Tzimas, P., 2007. Protection of volatiles in a wine with low sulfur dioxide by caffeic acid or glutathione. Am. J. Enol Vitic. 58, 274-278.

Singleton, V.L., 1987. Oxygen with phenols and related reactions in musts, wines, and model systems: Observations and practical implications. Am. J. Enol. Vitic. 38, 69-77.

Tao, Y.-S. \& Li, H., 2009. Active volatiles of Cabernet Sauvignon wine from Changli County. Natural Science 1, 176-182.

Vaimakis, V. \& Roussis, I.G., 1996. Must oxygenation together with glutathione addition in the oxidation of white wine. Food Chem. 57, 419422.

Vas, G. \& Vekey, K., 2004. Solid-phase microextraction: A powerful sample preparation tool prior to mass spectrometric analysis. J. Mass Spectrom. $39,233-254$.

Welke, J.E., Manfroi, V., Zanus, M., Lazarotto, M. \& Alcaraz-Zini, C. 2012. Characterization of the volatile profile of Brazilian Merlot wines through comprehensive two dimensional gas chromatography time-of-flight mass spectrometric detection. J. Chromatogr. A 1226, 124-139. 